\title{
MALAT1 regulates the transcriptional and translational levels of proto-oncogene RUNX2 in colorectal cancer metastasis
}

\author{
Qing Ji', Guoxiang Cai ${ }^{2}$ Xuan Liu', Yi Zhang ${ }^{1}$, Yan Wang ${ }^{1}$, Lihong Zhou' ${ }^{1}$, Hua Sui ${ }^{1}$ and Qi Li ${ }^{1}$
}

\begin{abstract}
Ectopic expression of IncRNA-MALAT1 has been discovered in recurrent colorectal cancer (CRC) and metastatic sites in postsurgical patients, however, its biological mechanism remained unelucidated. Our study first revealed the novel roles of MALAT1 in promoting CRC metastasis through two mechanisms: first, MALAT1 binds miR-15 family members, to "de-inhibit" their effect on LRP6 expression, enhances $\beta$-catenin signaling, leading to elevated transcriptional levels of downstream target genes RUNX2. Second, MALAT1 binds SFPQ, and dissociates SFPQ/PTBP2 dimer to release free PTBP2, which elevates translational levels of RUNX2, through interacting with IRES domain in the $5^{\prime} U T R$ of the corresponding RUNX2 mRNAs. Moreover, increased RUNX2 expression levels were detected in recurrent CRC tumors, which were closely associated with TMN stages, metastasis, as well as CRC patients' survival. Our study demonstrated that MALAT1 and RUNX2 may serve as two biomarkers for predicting the recurrence and metastasis of CRC patients.
\end{abstract}

\section{Introduction}

Colorectal cancer (CRC) is one of the leading causes of cancer-related deaths worldwide. Despite various clinical advances in systematic therapies for CRC patients, the 5year survival rate remains unsatisfactory ${ }^{1}$. Many advanced CRC patients died due to the distant metastases rather than primary tumor. Recurrence and metastasis after tumor resection have been a problem in tumor prognosis and therapy ${ }^{2}$. However, the complicated molecular mechanisms involved in CRC metastasis remain to be further investigated.

Long noncoding RNAs (lncRNAs), non-protein-coding transcripts longer than 200 nucleotides, regulate the expression levels of target genes through multiple mechanisms ${ }^{3}$. In recent years, several lncRNAs have been found to play vital roles in regulating CRC development

\footnotetext{
Correspondence: Qi Li (Lzwf@hotmail.com)

'Department of Medical Oncology, Shuguang Hospital, Shanghai University of Traditional Chinese Medicine, 201203 Shanghai, China

${ }^{2}$ Department of Colorectal Surgery, Fudan University Shanghai Cancer Center, 200032 Shanghai, China

These authors contributed equally: Qing Ji, Guoxiang Cai, Xuan Liu

Edited by A. Peschiaroli
}

and metastasis ${ }^{4,5}$. Metastasis associated in lung adenocarcinoma transcript 1 (MALAT1) is a recently characterized lncRNA, located on human chromosome 11q13, spanning about $8708 \mathrm{bp}$, and lack of a clear open-reading frame thereof does not translate the protein product ${ }^{6}$. The discovered functions of MALAT1 relate to pre-mRNA processing $^{7,8}$, gene regulation ${ }^{9-11}$, and generation of 60 -nt tRNA-like short ncRNAs ${ }^{12}$. We previously reported that MALAT1 correlated with CRC metastasis, prognosis, and survival rate ${ }^{13,14}$. Moreover, it was found to regulate CRC metastasis via a defined mechanism involving activation of $\beta$-catenin signaling and dissociation of SFPQ (PTB-associated splicing factor)/PTBP2 (polypyrimidine tract binding protein) complex ${ }^{13,15}$.

Several recent studies have reported the potential role of MALAT1 as a competing endogenous RNA (ceRNA) ${ }^{16-20}$ in different diseases; however, the mechanism of MALAT1 in CRC metastasis is not well defined. Our current studies uncovered a novel ceRNA role of MALAT1 via regulating transcriptional and translational levels of the proto-oncogene RUNX2 (Runt-related transcription factor 2) in CRC metastasis. 


\section{Results}

\section{CRISPR/Cas9-mediated MALAT1 knockout elevates the levels of miR-15 family}

Using RNA-sequencing technology, we found a significantly differential lncRNA expression pattern in postsurgical, recurrent primary, and metastatic sites, compared with primary sites of non-recurrent CRC patients (Fig. 1a, Supplementary Data S1). As one of the differentially expressed lncRNAs, MALAT1 had higher expression levels in recurrent primary and metastatic sites, relative to non-recurrent primary tumors (Fig. 1b), and this result was validated by real-time PCR in 124 CRC primary and metastatic tissues (Fig. 1c). In addition, we discovered that CRC patients with lower MALAT1 expression in primary tumors had better prognosis with longer OS (overall survival) and DFS (disease-free survival) (Fig. 1d, e). ROC curve analysis further supported the potential diagnostic value of MALAT1 in CRC metastasis (Fig. 1f).
We previously reported that MALAT1 promoted CRC metastasis via a preliminarily elucidated mechanism ${ }^{13,15}$. To thoroughly understand the biological mechanisms, we first genomically knocked out MALAT1 gene using CRISPR/Cas9 technology. Using the online guiding RNA design software (https://zlab.bio/guide-design-resources), two optimal CRISPR nuclease sgRNAs targeted to the exon region between 814 and 1557 nt of MALAT1 genome were designed (Fig. 2a), subcloned into the PX462 plasmid (Supplementary fig. 1a), and were validated effectively in HEK293T cells (Fig. 2b). To increase the efficiency of targeted loss-of-function pool screens, the above-mentioned validated oligonucleotides targeting MALAT1 were subcloned into LentiCRISPRv2 (Supplementary fig. 1b). Through a series of screening experiments (Supplementary fig. 1c), the MALAT1 that stably knocked out LoVo cells originated from clone 4 (M4) was obtained and named LoVo/MALAT1 ${ }^{-/-}$(Fig. 2c,

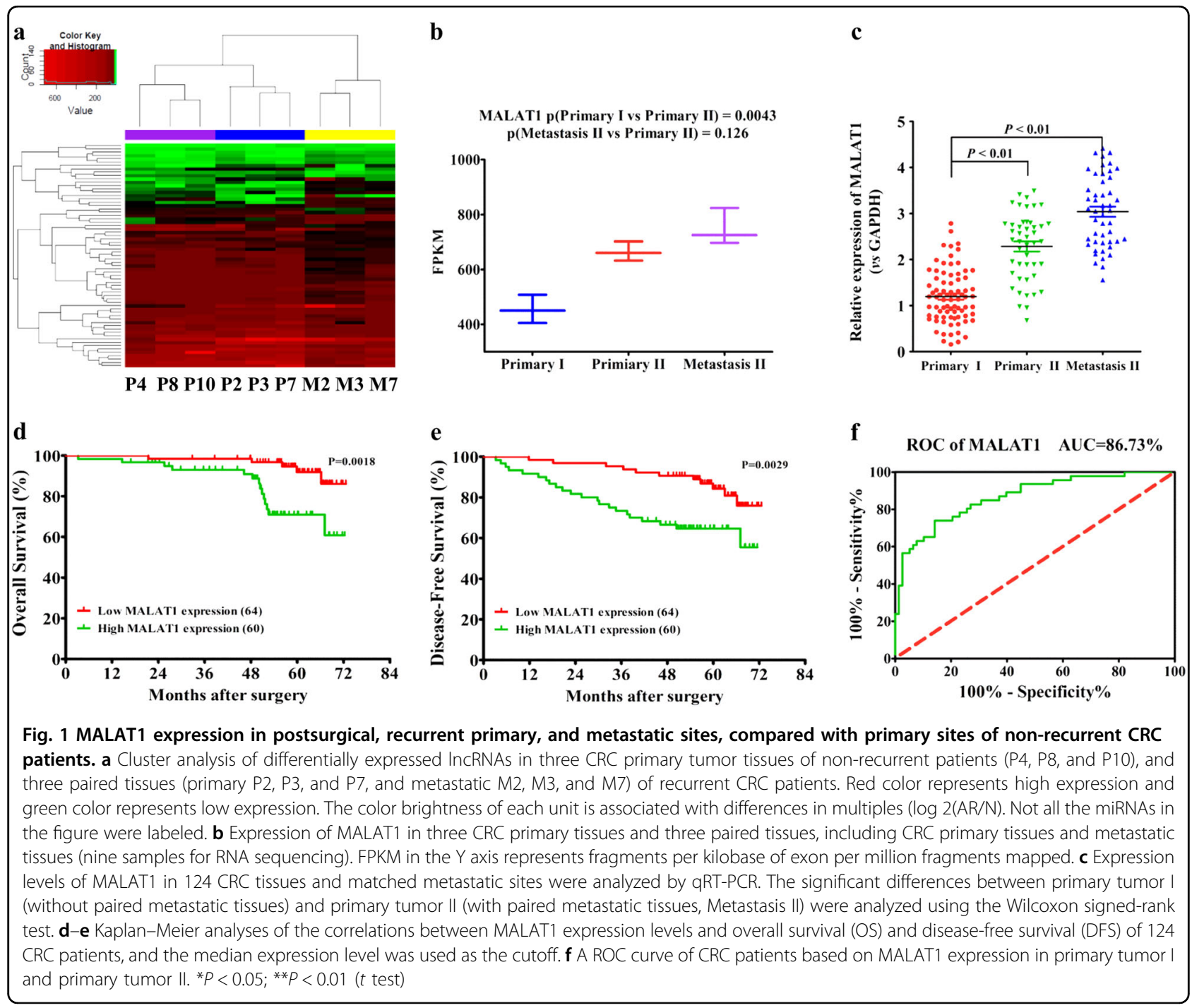




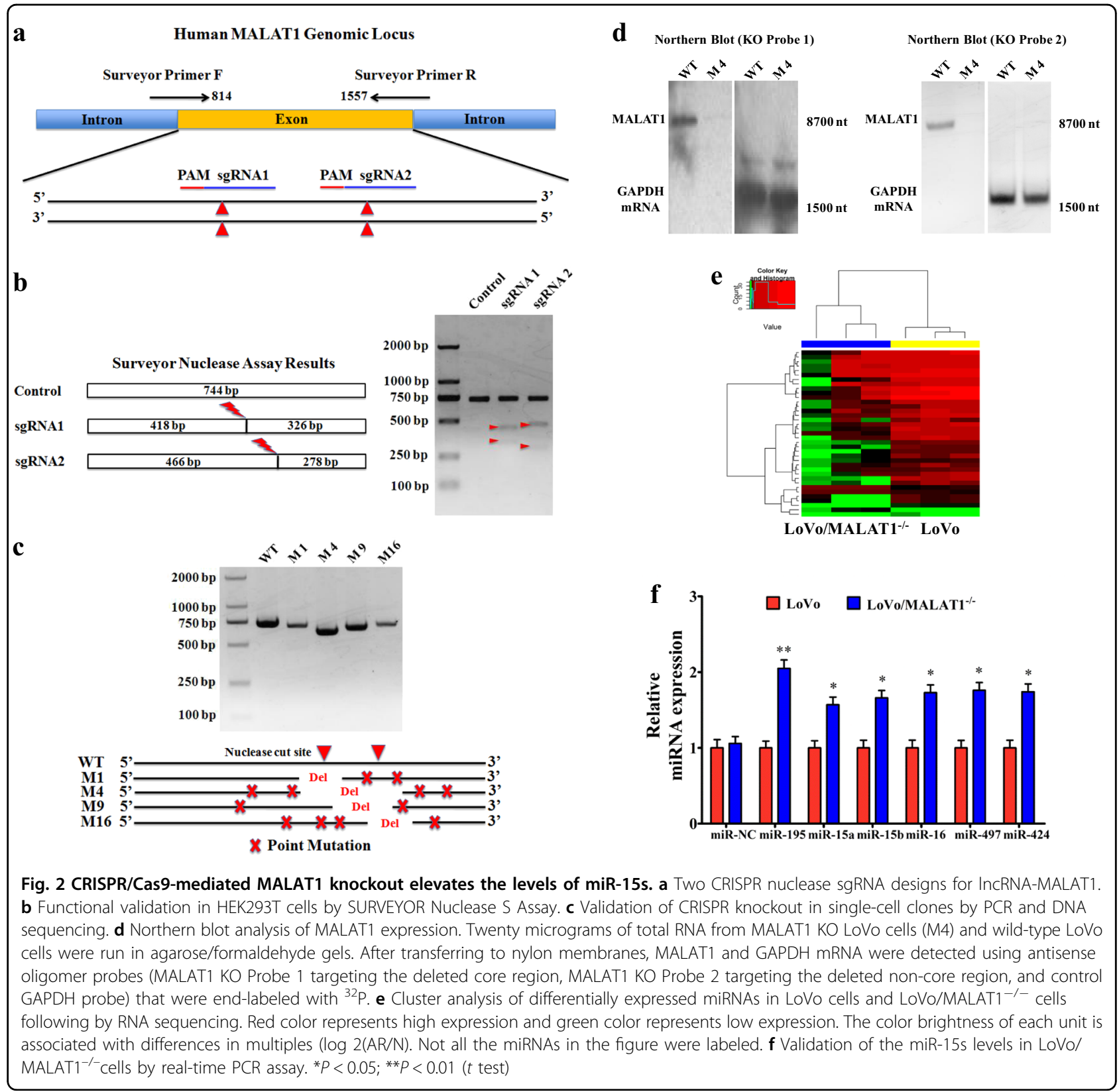

Supplementary Data S2 and Supplementary fig. 1d). Northern blotting analysis further confirmed the specific deletion executed by CRISPR/Cas9 sgRNA. In detail, using the MALAT1 KO Probe 1 targeting the deleted core region (1198-1218 nt) and MALAT1 KO Probe 2 targeting the non-deleted core region (838-857 nt), we could obtain the targeted fragment (full-length MALAT1 fragment, $8708 \mathrm{nt}$ ) in wild-type LoVo cells, but not in the M4 cells (Fig. 2d). Furthermore, in Supplementary fig. 2, the results confirmed that we have successfully realized the knockout of MALAT1 gene in CRC cells.

Next, to find the potential targets of MALAT1, a large number of differentially expressed miRNAs were found by
RNA sequencing, and some of them were validated by real-time PCR. Interestingly, the levels of the miR-15 family members (miR-15s) in LoVo/MALAT1 ${ }^{-/-}$cells increased significantly (Fig. 2e, f), suggesting that MALAT1 knockout elevated the levels of miR-15s. However, the biological mechanism of MALAT1 regulating miR-15s levels remained to be defined.

\section{MALAT1 functions as a ceRNA regulating miR-15 family}

Currently, numerous lncRNAs or mRNAs have been discovered to function as ceRNAs ${ }^{21}$, through binding to miRNAs and regulating their expression levels. By bioinformatics analysis, we constructed the interaction 


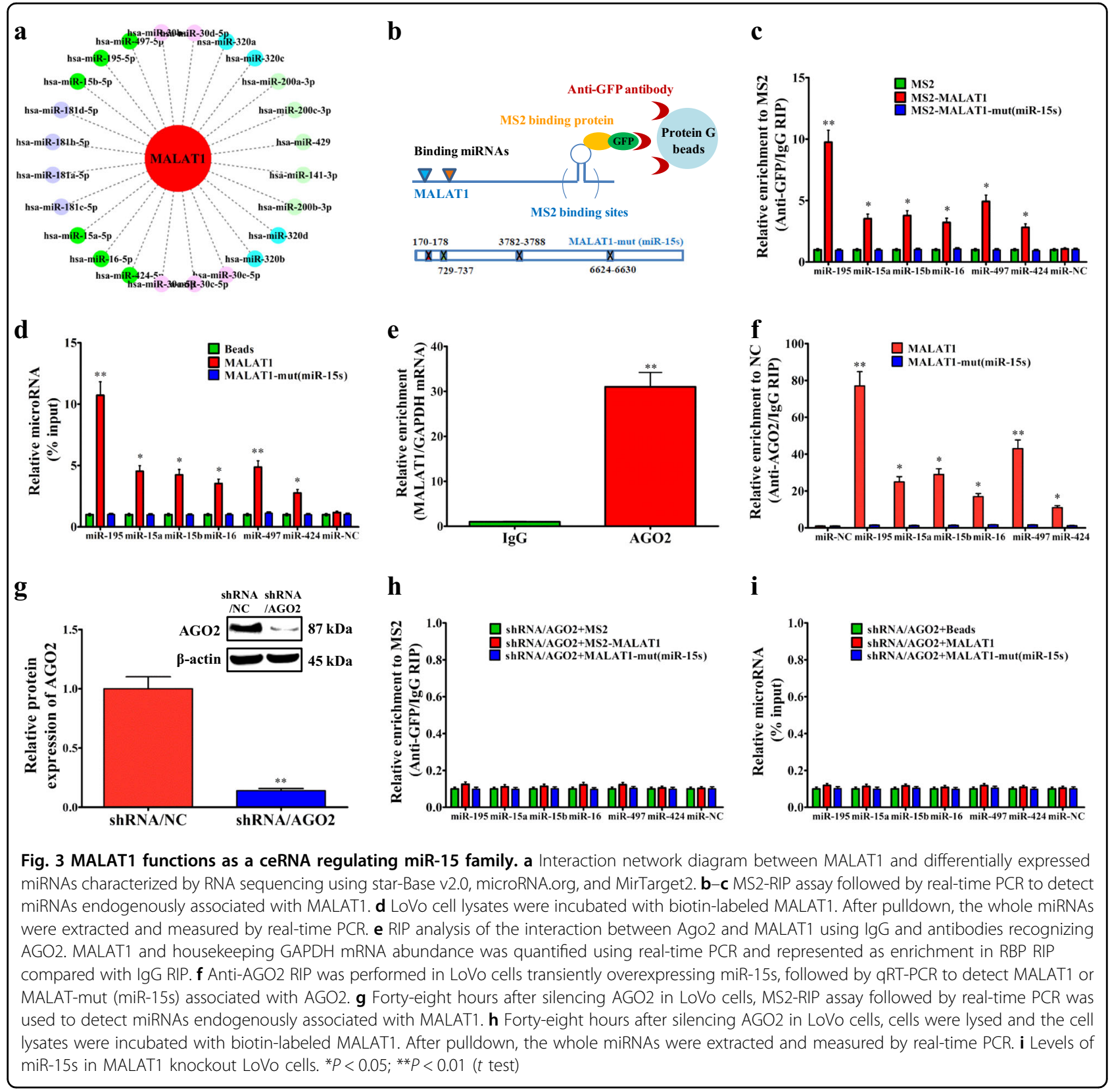

network diagram between MALAT1 and closely associated miRNAs, and emphatically labeled the miR-15 family (miR-15s) (Fig. 3a). The miR-15s, including miR15a, miR-15b, miR-16, miR-195, miR-424, and miR-497, had at least four MALAT1 interaction regions, two of which were proximal (Supplementary fig. 3a, b). To validate the direct binding between MALAT1 and miR-15s at endogenous levels, we performed RIP experiment to pull down the endogenous MALAT1-associated miRNAs, and demonstrated via qPCR that the MALAT1 RIP in LoVo cells was significantly enriched for miR-15s compared with the empty vector (MS2), IgG, non-targeting miRNA
(miR-67), and MALAT1-mut (miR-15s) (Fig. 3b-c). To further confirm the specific interaction between MALAT1 and miR-15s, we in vitro transcribed and biotin labeled MALAT1, and successfully pulled down the miR$15 \mathrm{~s}$ (Fig. 3d). In addition, the dual-luciferase reporter assay in Supplementary fig. $3 \mathrm{c}-\mathrm{f}$ further validated the specific association between MALAT1 and miR-15s.

Generally, miRNAs bind to the 3'UTR of target mRNA, to repress translation, or promote degradation through Argonaute2 $(\mathrm{AGO} 2)^{22}$. Due to AGO2 protein, as well as other proteins such as TNRC6A and/or importin, mature miRNAs can be transported to the nucleus from the 
cytoplasm $^{23-26}$. Using nuclear extracts and the AGO2 antibody, we confirmed the interaction between AGO2 and MALAT1 (Fig. 3e). Furthermore, in Fig. 3f, our results showed that endogenous MALAT1 pulldown by AGO2 was specifically enriched in miR-15s-transfected LoVo cells, indicating that miR-15s are bona fide MALAT1 targeting miRNAs. This demonstrated that miR-15s bound to MALAT1 without affecting the degradation of MALAT1. In the presence of AGO2 protein, MALAT1 maintained stable interaction with miR15s. Therefore, we further tested the interaction in the absence of AGO2 protein via AGO2 knockdown (Supplementary fig. $3 \mathrm{~g}$ and Fig. $3 \mathrm{~g}$ ), and found a negative interaction between MALAT1 and miR-15s (Fig. 3h, i).

In addition, not surprisingly, ectopically expressed MALAT1-WT, but not the MALAT1-mutant (miR-15s), reduced the levels of miR-15s (Supplementary fig. 3h). However, to function as a ceRNA, the abundance of MALAT1 and miR-15s should be comparable. We found that in LoVo cells, the expression levels of MALAT1 were approximately 250 copies per cell, and the mature miR-15s levels $\sim 115$ copies per cell. Overexpression of MALAT1WT upregulated the copies of MALAT1 and further downregulated the copies of miR-15s; however, overexpression of MALAT1-mut did not affect the copies of MALAT1 and miR-15s (Supplementary fig. 3i). Considering that the two fragments $(170-178 \mathrm{nt}$ and $730-737 \mathrm{nt}$ ) within the MALAT1 may be the miR-15s' seeding targets, we also designed two probes around the two fragments to confirm the success of MALAT1 knockout by CRISPR/Cas9 and exclude the effect of MALAT1 on miR-15s. The northern blotting results in Supplementary fig. 4a, b demonstrated that we could only obtain the targeted fragment (full-length MALAT1 fragment, $8708 \mathrm{nt}$ ) in wild-type LoVo cells, but not in the M4 cells, which excluded the direct binding between MALAT1 and miR-15s in MALAT1 ${ }^{-1-}$ CRC cells. All these data demonstrated that MALAT1 was physically associated with the miR-15s and may function as a ceRNA.

\section{miR-15s inhibits LRP6 expression and activation of downstream $\beta$-catenin signaling}

Our above-mentioned results aroused our interest in searching the downstream target genes of miR-15s. Bioinformatic analysis revealed several key targets of miR15s (Supplementary fig. 5a), including LRP6, one of the Wnt receptors, which play important roles in the activation of Wnt/ $\beta$-catenin signaling. Moreover, our previous research has suggested that MALAT1 could regulate $\beta$-catenin signaling pathway ${ }^{15}$, but through an undefined mechanism. In Supplementary fig. 5b-g, our results clearly interrogated the important role of miR-195 (a typical representative of miR-15s) in regulating target gene LRP6. In addition, activation of canonical Wnt/ $\beta$-catenin signaling requires Wnt ligands to bind to the membrane receptors such as LRP6, which in turn regulates GSK-3 $\beta$ phosphorylation and $\beta$-catenin accumulation in the nucleus, and then, the nuclear $\beta$-catenin and other transcription factors such as LEF, TCF, and BCL9 will trans-activate downstream target genes ${ }^{27}$. Our data in Supplementary fig. $5 f-i$ further illustrated a negative regulatory effect of miR-195 on LRP6 expression.

Moreover, in Supplementary fig. 6, by the in vivo and in vitro experiments, we further confirmed the regulatory function of miR-195 on CRC metastasis through LRP6. In addition, our qPCR results revealed high levels of miR-195 in recurrent primary and metastatic sites, and nonrecurrent primary CRC tissues, but with no statistical difference (Supplementary fig. 7a). Significantly higher LRP6 mRNA and protein levels were also observed in recurrent primary tumors and metastatic sites than those in the non-recurrent primary tumors, and the LRP6 levels were much higher in metastatic sites than those in recurrent primary tumors (Supplementary fig. 7b, c).

\section{MALAT1 regulates RUNX2 transcription via miR-15s/LRP6/ $\beta$-catenin signaling pathway}

The above results showed the regulatory effect of MALAT1 on miR-15s/LRP6 (Fig. 4a). Furthermore, we found that MALAT1 knockout decreased the LRP6 3' UTR reporter activity significantly, while overexpressing full length of MALAT1 in LoVo/MALAT1 ${ }^{-1-}$ cells could largely rescue the LRP6 3'UTR reporter activity (Fig. 4b). Moreover, MALAT1 knockout significantly reduced the mRNA expression of LRP6, while overexpressing full length of MALAT1 in LoVo/MALAT1 ${ }^{-1-}$ cells rescued the mRNA levels of LRP6 (Fig. 4c).

Due to the important roles of LRP6 in the activation of $\mathrm{Wnt} / \beta$-catenin signaling pathway, we further verified the regulating effect of MALAT1 on $\beta$-catenin signaling pathway. The inhibitor for $\beta$-catenin signaling pathway, XAV $939^{28}$, was used to block the activation of $\beta$-catenin signaling pathway. Knockout of MALAT1 could significantly decrease the nuclear accumulation of $\beta$-catenin, while overexpression of MALAT1 could effectively reverse the distribution of $\beta$-catenin in the nucleus (Fig. $4 \mathrm{~d})$. However, when the LoVo/MALAT1 ${ }^{-1-}$ cells were pretreated with XAV939, overexpression of MALAT1 had little effect on $\beta$-catenin nuclear translocation (Fig. 4d), which was further validated by the immunofluorescent staining results (Fig. 4e, f). Moreover, the luciferase reporter assay further confirmed the regulating effect of MALAT1 on the activity of LEF/TCF promoter (Fig. 4g). In addition, in Supplementary fig. 8, we further confirmed the necessity of miR-15s and LRP6 in MALAT1 enhancing $\beta$-catenin signaling.

Since the alteration of nuclear $\beta$-catenin could greatly affect its downstream target gene transcription, we next 


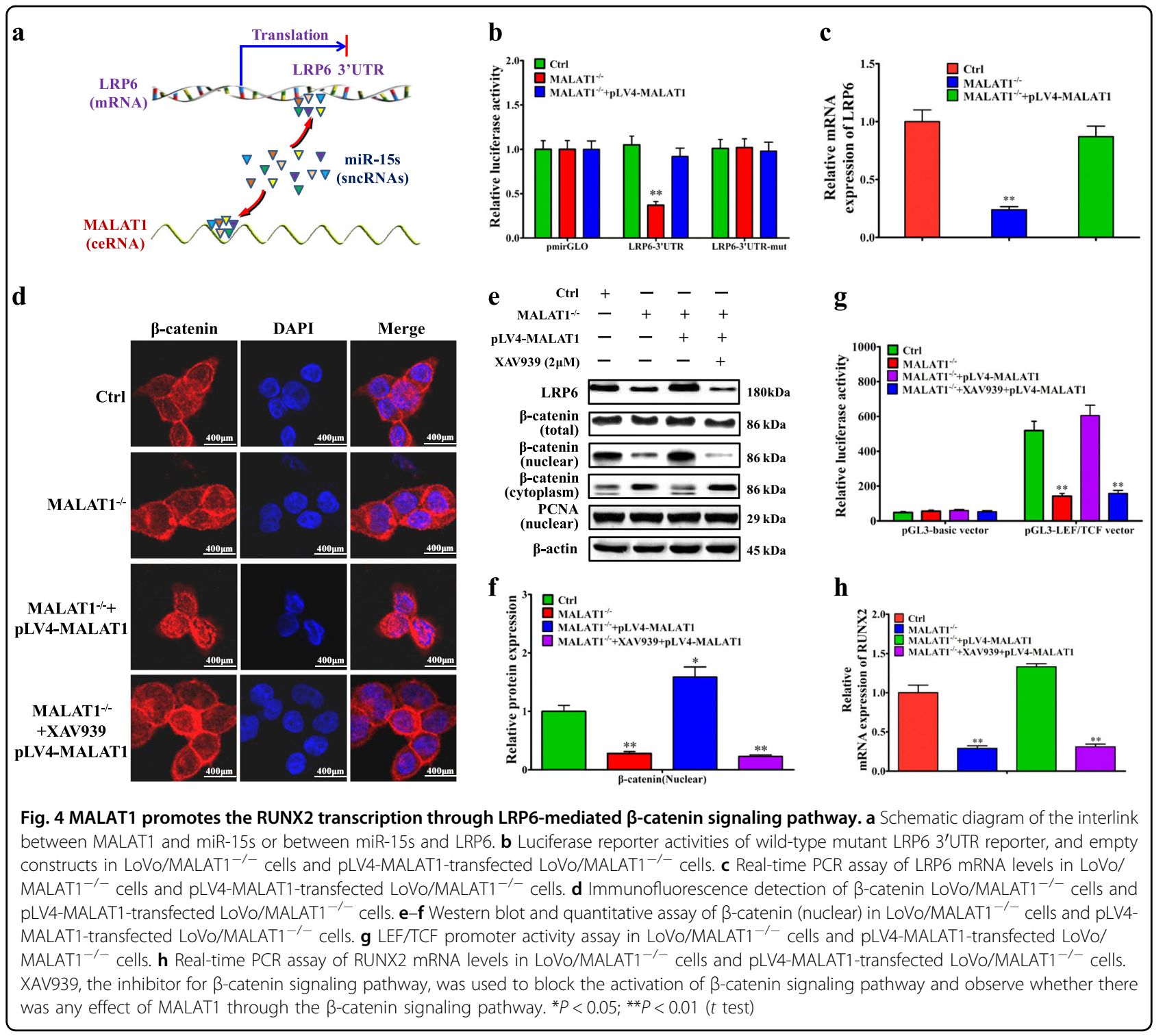

performed RNA sequencing to observe the mRNA expression profile differences in LoVo and LoVo/ MALAT1 $1^{-/-}$cells (Supplementary fig. 9a). Several $\beta$-catenin-associated mRNAs were found to be downregulated in LoVo/MALAT1 ${ }^{-1-}$ cells, such as $H I F-1 \alpha$, $V E G F a, C O X-2, c-M y c, C C N D 1, R U N X 2$, and $M M P-7$ (Supplementary fig. 9b). RUNX2, a key transcription factor encoded by the RUNX2 gene, drew our extensive attention. Several reports have found the important roles of RUNX2 in various tumors ${ }^{29,30}$. As shown in Supplementary fig. 9c, MALAT1 knockout reduced the mRNA expression of RUNX2, VEGFa, and COX-2 significantly, while overexpressing full length of MALAT1 in LoVo/ MALAT1 $^{-/-}$cells greatly rescued the mRNA levels of RUNX2, VEGFa, and COX-2, suggesting that MALAT1 significantly promoted the transcriptional levels of RUNX2. Most important of all, the existence of XAV939 significantly blocked the positive regulating effect of MALAT1 on the mRNA expression of RUNX2 (Fig. 4h), providing the direct evidence that the transcription of RUNX2 could be positively regulated by MALAT1. In addition, in Supplementary fig. 10, we also eliminated the influence of MALAT1 on other upstream ligands or receptors of $\mathrm{Wnt} / \beta$-catenin signaling pathway.

\section{MALAT1-mediated PTBP2 promotes the translation of RUNX2}

We previously reported that MALAT1 promoted CRC metastasis via increasing free PTBP2 protein $^{12}$, and higher mRNA and protein levels of PTBP2 were found in the recurrent tumors and lung/liver metastasis, than the ones in the non-recurrent tumors (Supplementary fig. 11a, b). 


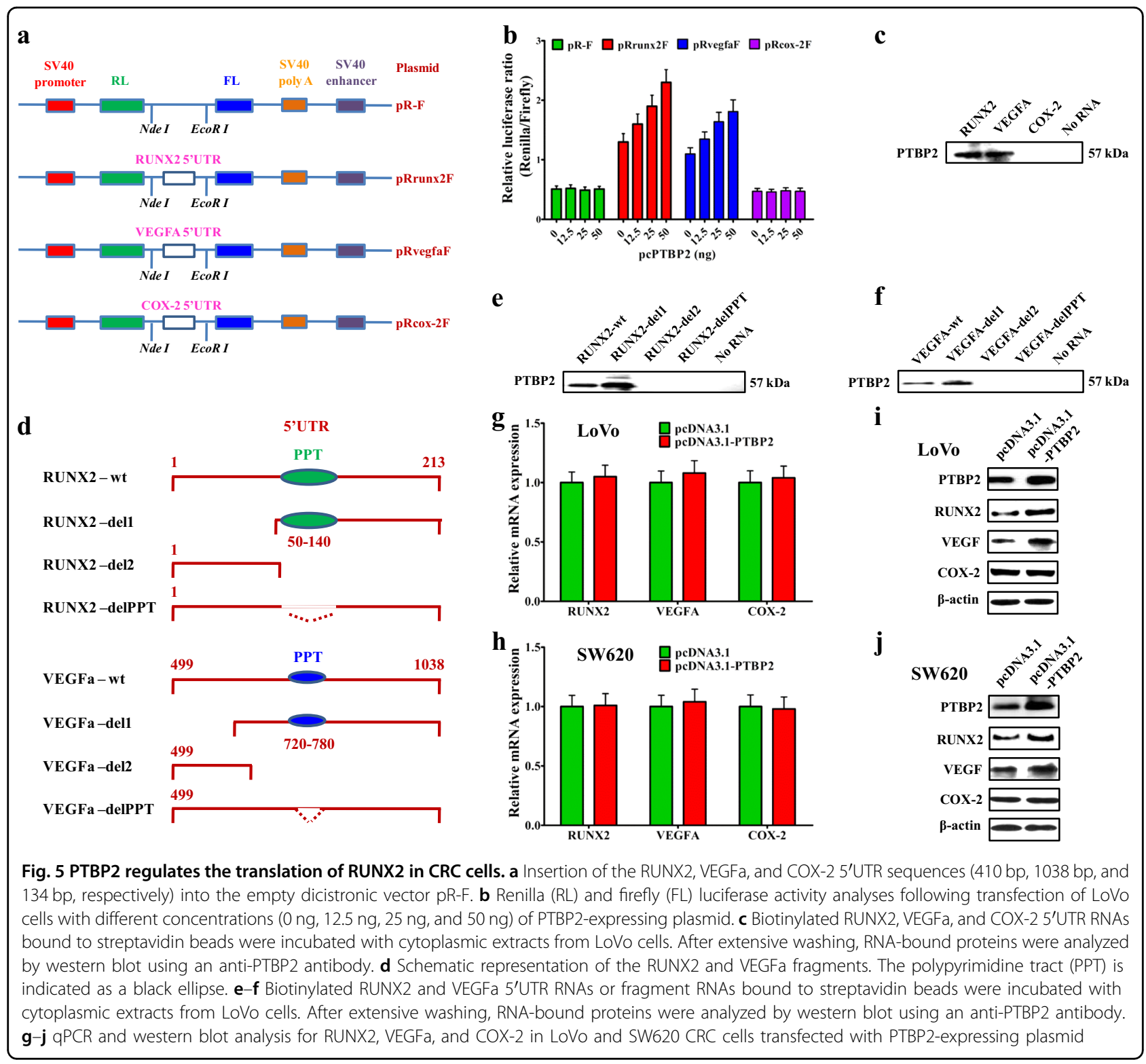

However, the downstream target genes of PTBP2 remained undefined. Some reports have demonstrated that PTBP2 bound to the 5'UTR-IRES domain of targeted mRNA to promote translation ${ }^{31,32}$. It is known that the $5^{\prime}$ UTRs of HIF1- $\alpha$, c-Myc, and VEGFa mRNA have IRES domains which are bound by PTBP2 promoting translation $^{33-35}$. Such phenomenon was not reported for RUNX2 mRNA. We analyzed the 5'UTR sequence of RUNX2 mRNA and found potential IRES domain, especially the PPT (polypyrimidine tract) sequences, whose secondary structure was high similar to VEGFa's (Supplementary fig. 12a-d). To confirm our hypothesis, we generated dual-luciferase reporter plasmid containing the 5'UTR-IRES region of RUNX2 (Fig. 5a), and overexpression of PTBP2 dramatically increased luciferase activity of RUNX2 and VEGFa reporter plasmids in a dose-dependent manner (Fig. 5b).

Urged by the above data, we next studied if PTBP2 could bind to the IRES domain of RUNX2. In vitro-synthesized, biotin-labeled mRNAs containing the IRES domains of RUNX2, VEGFa, and COX-2 were mixed with LoVo cell lysate, respectively, and the mRNA-binding proteins were pulled down using streptavidin beads. Western blot assay of the pulled-down proteins confirmed PTBP2 as a binding partner of IRES domains of RUNX2 and VEGFa mRNA, but not for the IRES domain minus COX-2 mRNA (Fig. 5c). To test whether the well-defined polypyrimidine tract (PPT) in IRES was critical for PTBP2 binding to the mRNAs of RUNX2 and VEGFa, we dissected the 5'UTR regions of RUNX2 and VEGFa into smaller fragments or 


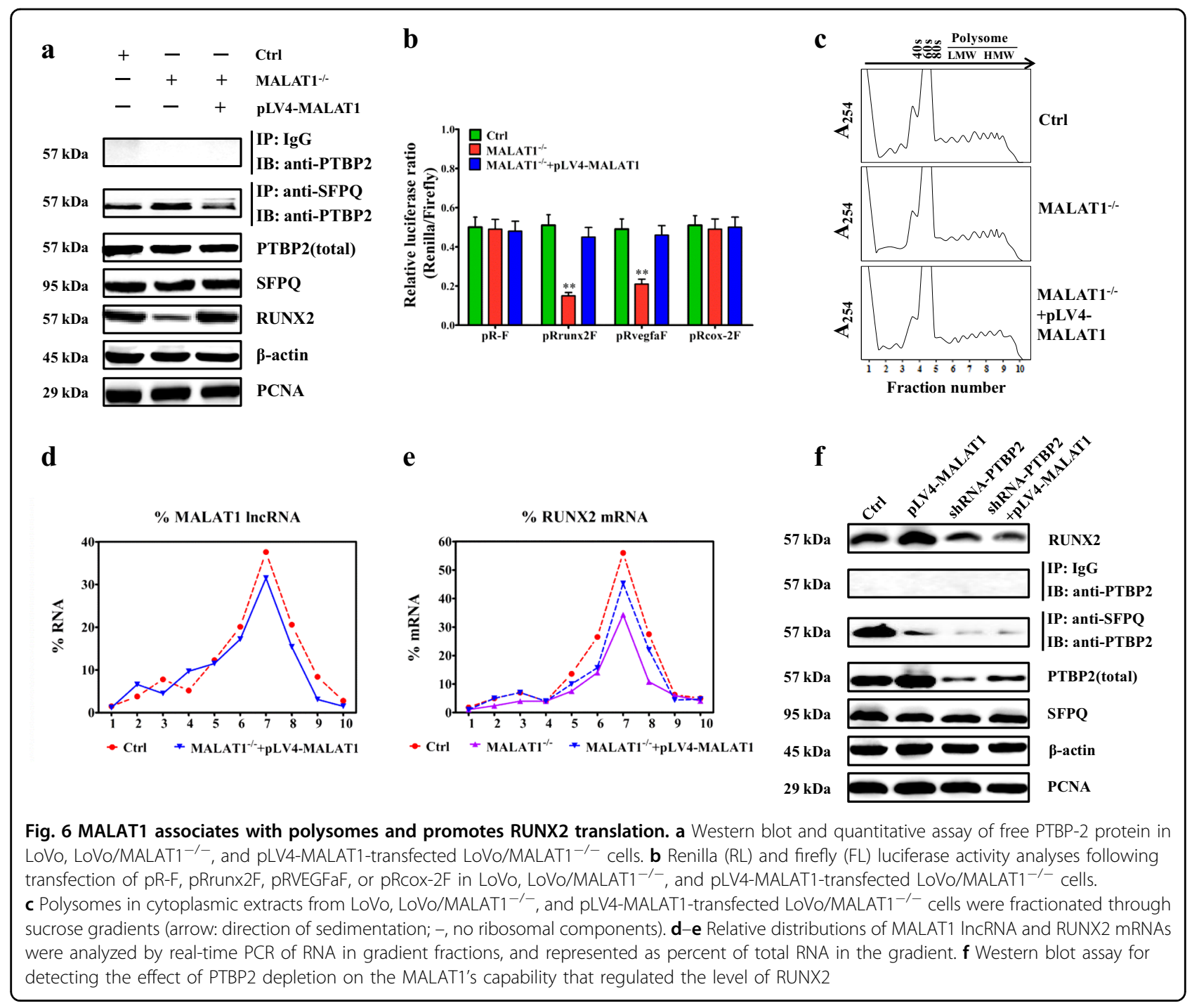

fragments with PPT deleted, and made biotin-labeled mRNA for PTBP2-binding assays (Fig. 5d). The results showed that PTBP2 could only bind to the mRNA fragments that harbored PPT, implying its critical role in mediating PTBP2 interaction with the IRES domains of RUNX2 and VEGFa mRNA (Fig. 5e, f). We also determined that PTBP2 overexpression did not affect the mRNA levels of RUNX2, VEGFa, and COX-2 (Fig. 5g, h), but enhanced protein levels of RUNX2 and VEGFa (Fig. 5i, j).

Subsequently, in Fig. 6a, b, the results showed that MALAT1 knockout increased the abundance of SFPQ/ PTBP2, lower amount of free PTBP2 proteins, while overexpressing full length of MALAT1 in LoVo/ MALAT $^{-1-}$ cells could largely rescue the amount of free PTBP2 proteins. Moreover, MALAT1 knockout decreased luciferase activity of RUNX2 and VEGFa reporter plasmids, except COX-2 reporter plasmids, while overexpressing full length of MALAT1 in LoVo/
MALAT1 $^{-1-}$ cells could largely rescue luciferase activities of RUNX2 and VEGFa reporter plasmids, but not COX-2 reporter plasmids (Fig. 6c).

Furthermore, to prove enhanced or reduced translation of RUNX2 and VEGFa in MALAT1 overexpressed or deleted cells, we have performed polysome profiling followed by RT-qPCR analyses, and the results were consistent with the dual-luciferase reporter analysis and western blot results. In detail, LoVo cell lysates expressing different MALAT1 levels were fractionated through sucrose gradients. Fractions 1 and 2 were the lightest components, fractions 3,4 , and 5 were the relatively large ribosomal subunits (40S, 60s) and monosomes (80S), and fractions 6-10 were the progressively larger polysomes that ranged from low- to high-molecular weight (LMW, HMW) (Fig. 6d). MALAT1 knockout and/or MALAT1 overexpression did not change the polysome distribution profiling (Fig. 6d), indicating that these interventions did 

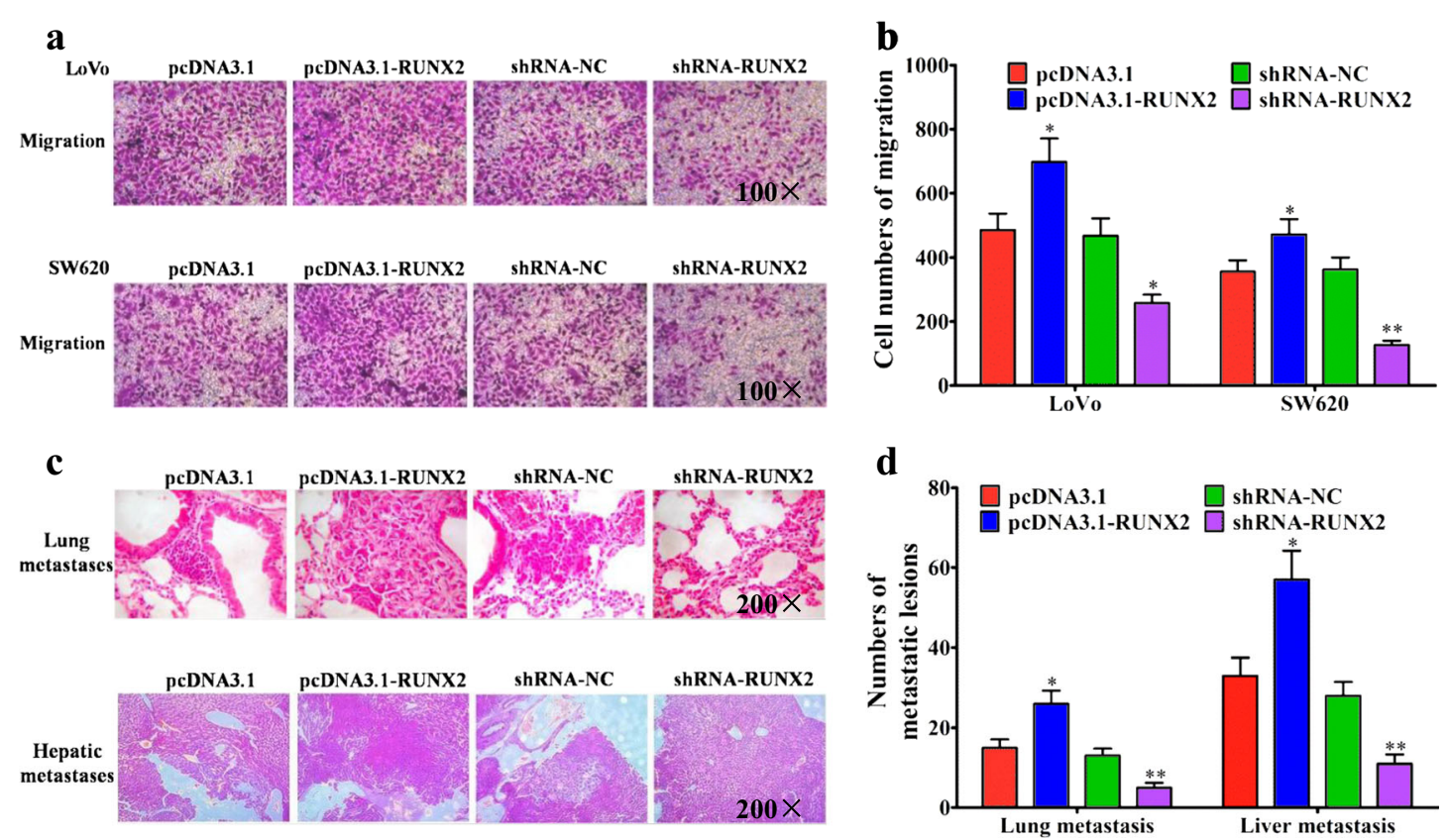

Fig. 7 RUNX2 promotes the metastasis of CRC cells in vitro and in vivo. a Migration assays of LoVo or SW620 cells transfected with pcDNA3.1, pcDNA3.1-RUNX2, shRNA-NC, and shRNA-RUNX2, respectively. b Numbers of migrated cells were shown as mean $\pm S D ; n=3$. c Representative results of H\&E staining of the metastatic nodules in the lung and liver from six mice subjected to the indicated treatments. $\mathbf{d}$ Quantification of lung and liver metastasis from six mice subjected to the indicated treatments. ${ }^{*} P<0.05$; ${ }^{* *} P<0.01$ ( $t$ test)

not affect global translation. After isolating RNA from each fraction, real-time PCR analysis indicated that MALAT1 was abundant in fractions 6-9 corresponding to LoVo and LoVo/MALAT1 ${ }^{-1-} /$ pLV4-MALAT1 (Fig. 6e). The polysomal sizes of RUNX2 and VEGFa mRNAs showed their reduced translation after MALAT1 knockout; however, MALAT1 overexpression totally prevented the reduction in translation of RUNX2 and VEGFa mRNAs (Fig. $6 \mathrm{f}$ and Supplementary fig. 13a). The distribution of the COX-2 mRNA and housekeeping GAPDH mRNA did not show the former pattern (Supplementary fig. 13b, c), indicating that MALAT1 knockout and/or MALAT1 overexpression specifically affected RUNX2 and VEGFa mRNAs. Further, a western blot experiment demonstrated that depletion of PTBP2 impaired the MALAT1's capability to enhance the level of RUNX2 through the release of PTBP2 (Fig. 6g). To sum up, the above-mentioned data clearly illustrated a positive effect of MALAT1 and PTBP2 on the translation of RUNX2. In addition, we confirmed in Supplementary fig. 14 that the above two pathways act in an independent manner.

\section{RUNX2 overexpression correlates with human CRC progression}

RUNX2 was reported to affect the metastasis of gastric cancer $^{29}$, prostate cancer ${ }^{30}$, etc., but its function in CRC remained unknown. Therefore, we overexpressed or
shRNA silenced RUNX2 gene in LoVo and SW620 cells, and found a positive role of RUNX2 in promoting migration of CRC cells (Fig. 7a, b). In the in vivo mouse models, overexpressing of $R U N X 2$ promoted the lung and liver metastasis of CRC cells, while silencing of RUNX2 did the contrary (Fig. 7c, d). All these experiment results indicated the promoting effect of RUNX2 in CRC metastasis.

Based on the above-mentioned results, the mRNA and protein expression levels of RUNX2 were further detected in the above 124 CRC tumor specimens. The results demonstrated that the mRNA levels of $R U N X 2$ were significantly higher in recurrent tumors and metastatic sites than those of non-recurrent ones (Fig. 8a), and the mRNA levels were closely associated with CRC TMN stages and liver/lung metastasis (Table 1). Survival analysis concluded that CRC patients with lower RUNX2 mRNA expression had longer OS and DFS (Fig. 8b, c). Immunohistochemistry staining revealed significantly higher protein levels of RUNX2 in recurrent tumors and lung/liver metastasis than the ones in non-recurrent tumors (Fig. 8d), implying that RUNX2 overexpression correlated tightly with CRC progression.

\section{Discussion}

Numerous studies have uncovered abnormal expression of lncRNAs in primary and metastatic tumor sites, 


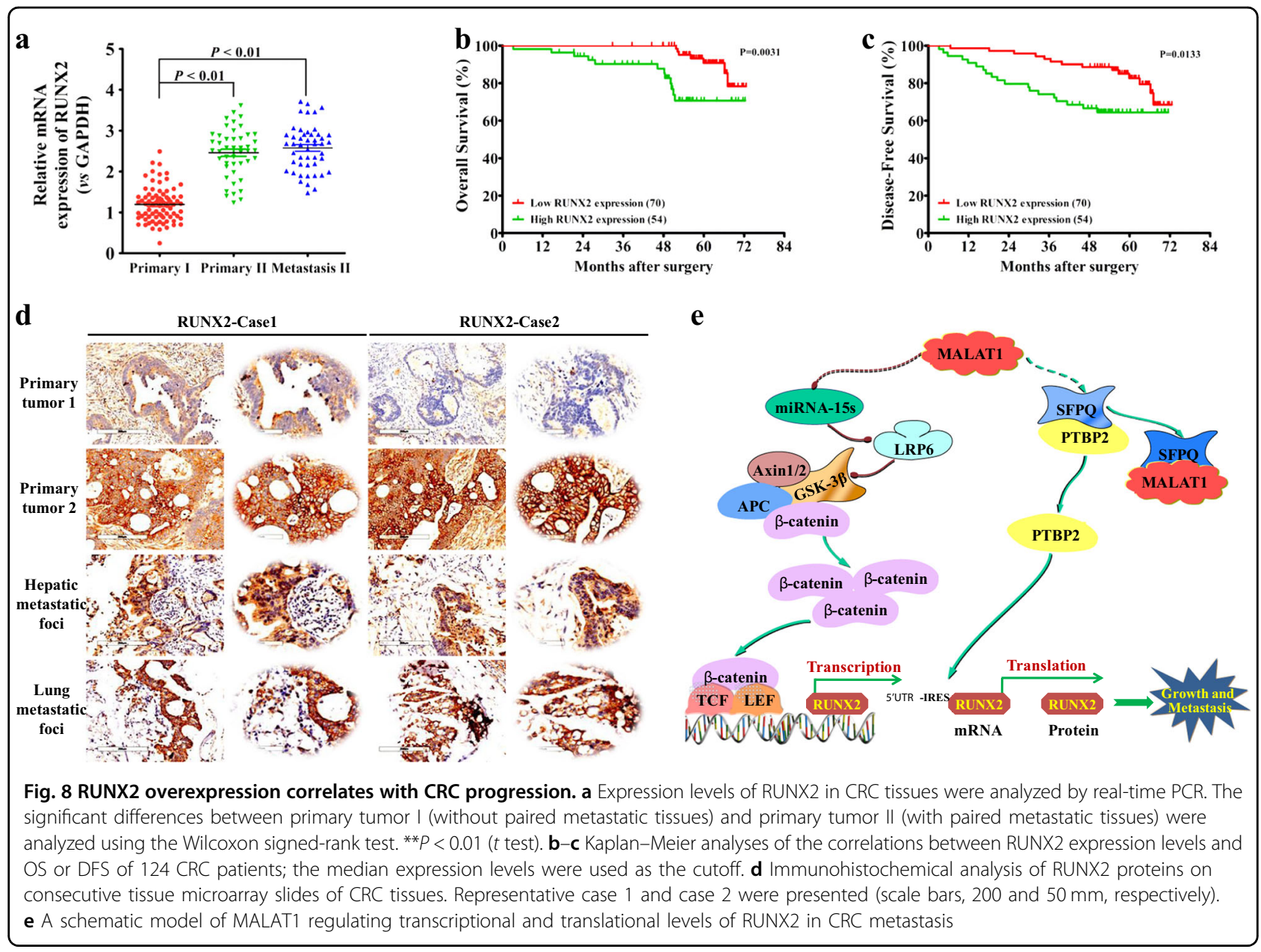

suggesting their significant roles of oncogenes or tumor suppressors $^{36,37}$. As an important lncRNA, MALAT1 has been described as a metastasis-promoting gene in various cancers ${ }^{6,10,11,13,18-20}$. However, in the study by Kim et al., the authors showed that targeted inactivation of the MALAT1 gene in a transgenic mouse model of breast cancer, without altering the expression of its adjacent genes (Frmd8, Neat1, etc.), promoted lung metastasis ${ }^{38}$. In our study, we found that MALAT1 had a significantly higher expression in recurrent CRC primary and metastatic tumors in comparison with the non-recurrent primary tumors. The CRC patients with a higher level of MALAT1 in primary tumors had poor prognosis.

Previous studies implied a close link between MALAT1 and $\beta$-catenin signaling, but the detailed mechanism remained elusive ${ }^{15}$. Here, we uncovered a novel ceRNA role of MALAT1 indirectly modulating $\mathrm{Wnt} / \beta$-catenin signal cascade. In a totally new manner, ceRNAs, including a pool of mRNAs, lncRNAs, and transcribed pseudogenes, bind miRNAs via miRNA response elements (MRE) to derepress miRNA target genes ${ }^{39}$. Our results elucidated that MALAT1 functioned as a ceRNA, by binding the miR-15s, specifically miR-195, to interfere with their inhibitory effect on expression of target genes.

Through bioinformatic prediction and scientific experiments, we discovered that, as the representative of miR-15s, miR-195 repressed the expression of LRP6, a key mediator of Wnt/ $\beta$-catenin signaling. LRP6, as a critical membrane receptor of the Wnt ligand, modulates tetramer formation of GSK-3 $\beta / \beta$-catenin/axin/APC, and regulates $\beta$-catenin nuclear translocation ${ }^{40}$. Li et al. described LRP6 stably expressed in HT1080 cells, increased the cytosolic $\beta$-catenin level, and enhanced cell proliferation $^{41}$. Liu et al. demonstrated that LRP6 overexpressed in human breast cancers, and the activation of Wnt signaling by LRP6 overexpression was sufficient to induce cancer formation $^{42,43}$. These studies highlighted the importance of LRP6 in Wnt-driven carcinogenesis. Our group discovered that miR-195 inhibited the LRP6 expression via binding to the 3'UTR of LRP6 mRNA, downregulated the nuclear translocation of $\beta$-catenin, and accordingly repressed the transcription of $\beta$-catenin downstream target genes, such as RUNX2 and VEGFa, which in turn caused lowered CRC metastasis. 
Table 1 Association between RUNX2 expression and clinicopathological variables of the studied CRC patients

\begin{tabular}{llll}
\hline Variables & Low RUNX2 & High RUNX2 & $P$ \\
& expression $(n=73)$ & expression $(n=51)$ \\
& $n(\%)$ & $n(\%)$
\end{tabular}

\begin{tabular}{|c|c|c|c|}
\hline \multicolumn{4}{|l|}{ Age } \\
\hline$>65$ & 16 (21.92) & 7 (13.73) & \multirow[t]{2}{*}{0.3481} \\
\hline$\leq 65$ & 57 (78.08) & $44(86.27)$ & \\
\hline \multicolumn{4}{|l|}{ Gender } \\
\hline Male & $41(56.16)$ & $37(72.55)$ & \multirow[t]{2}{*}{0.0894} \\
\hline Female & $32(43.84)$ & $14(27.45)$ & \\
\hline \multicolumn{4}{|l|}{ Tumor site } \\
\hline Rectum & $40(54.79)$ & $33(64.71)$ & \multirow[t]{2}{*}{0.3543} \\
\hline Colon & $33(45.21)$ & $18(35.29)$ & \\
\hline \multicolumn{4}{|c|}{$\begin{array}{l}\text { Tumor } \\
\text { differentiation }\end{array}$} \\
\hline Well & $18(24.66)$ & $21(41.18)$ & \multirow[t]{2}{*}{0.0761} \\
\hline \multirow{2}{*}{\multicolumn{4}{|c|}{$\begin{array}{l}\text { Moderate + } \\
\text { poor }\end{array}$}} \\
\hline & & & \\
\hline Stage II & $37(50.68)$ & $10(19.61)$ & \multirow[t]{2}{*}{0.0006} \\
\hline Stage III & $36(49.32)$ & 41 (80.39) & \\
\hline \multicolumn{4}{|c|}{$\begin{array}{l}\text { Lymph vascular } \\
\text { invasion }\end{array}$} \\
\hline Positive & $48(65.75)$ & $38(74.51)$ & \multirow[t]{2}{*}{0.3282} \\
\hline Negative & $25(34.25)$ & $13(25.49)$ & \\
\hline \multicolumn{4}{|l|}{$\begin{array}{l}\text { Perineural } \\
\text { invasion }\end{array}$} \\
\hline Positive & $56(76.71)$ & $42(82.35)$ & \multirow[t]{2}{*}{0.8301} \\
\hline Negative & 17 (23.29) & 9 (17.65) & \\
\hline \multicolumn{4}{|c|}{ Liver metastasis } \\
\hline Positive & $10(13.70)$ & $21(41.18)$ & \multirow[t]{2}{*}{0.0007} \\
\hline Negative & $63(86.30)$ & $30(58.82)$ & \\
\hline \multicolumn{4}{|c|}{ Lung metastasis } \\
\hline Positive & $1(1.37)$ & $8(15.69)$ & \multirow[t]{2}{*}{0.0035} \\
\hline Negative & 72 (98.63) & $43(84.31)$ & \\
\hline
\end{tabular}

The bold $P$ values are considered statistically significant

Our previous work has described that, MALAT1 competitively interacts with SFPQ protein, to disrupt the SFPQ/PTBP2 heterodimer formation and release free PTBP2 proteins ${ }^{13}$. Nevertheless, how PTBP2 affects downstream signaling pathways remained undefined. Our current study provides a convincing answer: PTBP2 regulates downstream target genes $R U N X 2$ and $V E G F a$. VEGFa is an endothelial growth factor, well known to promote CRC initiation and progression, especially participating in tumor angiogenesis and metastasis ${ }^{44,45}$. Nevertheless, the biological function of RUNX2 in CRC was little known. Sequence and structure analysis of RUNX2 yielded the IRES domain in its 5'UTR of mRNA. As evidence pointing out, the IRES domain is bound by numerous antagonizing factors, to modulate the translation process of the genes that harbor it ${ }^{46,47}$. We herein discovered that PTBP2 upregulated the translational levels of RUNX2 and VEGFa mRNA, by functioning on the $5^{\prime}$ UTR-IRES domains of their mRNAs. In contrary, for the IRES-null genes such as COX-2, PTBP2 had little effect on their translation process. These data suggested a specific functionality of PTBP2 toward the IRES domain and the translation of genes that have the IRES domain.

RUNX2 has been reported to affect the metastasis of gastric cancer ${ }^{29}$ and prostate cancer ${ }^{30}$. Our in vitro and in vivo studies also established a positive function for RUNX2 in promoting liver/lung metastasis of CRC cells. Analysis of CRC specimens further ascertained that RUNX2 mRNA and protein levels were distinctly higher in recurrent tumors (primary and metastatic) than the non-recurrent counterparts, and RUNX2 overexpression correlated tightly with CRC progression, especially the TMN stages and liver/lung metastasis. All these presented data suggested that RUNX2 might serve as a novel predictive and diagnostic biomarker for CRC metastasis.

In summary, we propose, as a ceRNA, that MALAT1 promotes CRC metastasis via at least two mechanisms. First, MALAT1 binds miR-15 family members, to "deinhibit" their effect on LRP6 expression, enhances $\beta$-catenin signaling, leading to elevated transcriptional levels of downstream target genes RUNX2. Second, MALAT1 binds SFPQ protein, dissociates SFPQ/PTBP2 dimer to release free PTBP2, which increases translational levels of RUNX2 mRNA, through interacting with the IRES domain in the $5^{\prime}$ UTR of the corresponding mRNAs (Fig. 8e).

\section{Materials and methods \\ Human tissues and cell lines}

A total of 78 human primary CRC tissues without metastasis and 46 human primary CRC tissues with matched hepatic or lung metastasis were collected between 2006 and 2015 at Shuguang Hospital, Shanghai University of Traditional Chinese Medicine, and Fudan University Shanghai Cancer Center. The general clinical information of all the 124 CRC patients was included in Supplementary Table 1. HEK293T cell line, human CRC cell lines LoVo and SW620, were purchased from American Type Culture Collection (ATCC). HEK293T, LoVo, and SW620 were cultured in Dulbecco's modified 
Eagle medium (DMEM), F12-K medium, and Leibovitz's L-15 medium, respectively. All the media were supplemented with $10 \%$ FBS (Gibco), $100 \mathrm{U} / \mathrm{mL}$ penicillin, and $100 \mathrm{mg} / \mathrm{mL}$ streptomycin. All the experimental procedures were approved by the Institutional Review Board of Shuguang Hospital, Shanghai University of Traditional Chinese Medicine.

\section{RNA sequencing and bioinformatics analysis}

CRC frozen tissues $(20 \mathrm{mg}$ each) were disrupted and homogenized, followed by RNA isolation (mirVana ${ }^{\mathrm{mm}}$ miRNA Isolation Kit, Thermo Fisher Scientific) in two fractions: small RNA ( $<200 \mathrm{nt})$, and large RNA ( $>200 \mathrm{nt})$. For small RNA sequencing (RNA-seq), small RNA libraries needed to be prepared first. Polyacrylamide gel electrophoresis (PAGE) was performed to separate the appropriate small RNAs (<40 nt). Thirty nanograms of the former small RNAs were modified with $3^{\prime}$ and $5^{\prime}$ adapters (Small RNA Library Prep Set for Illumina, NEB). Adapter-ligated RNAs were reverse-transcribed (ProtoScript ${ }^{\circledR}$ II First Strand cDNA Synthesis Kit, NEB) and amplified by PCR (Q5 High Fidelity Hot Start 2X Master Mix, NEB). Amplified libraries were size-selected by PAGE separation and sequenced (HiSeq2000, Illumina). For MACE, MACE libraries were first prepared as previously described ${ }^{48}$. Briefly, polyadenylated RNA was extracted (Poly (A) mRNA Magnetic Isolation Module, NEB) from large RNA fractions and reverse-transcribed (mRNA Second Strand Synthesis Module, NEB) with biotinylated poly $(\mathrm{dT})$ primers. cDNA was fragmented (E210 Covaris instrument, Covaris Inc., USA) to a 150-600-bp size range. Biotinylated ends were captured by streptavidin beads (Streptavidin Magnetic Beads, NEB) and ligated to modified adapters (mRNA Library Prep Reagent Set for Illumina). The MACE libraries were amplified by PCR (Q5 High Fidelity Hot Start 2X Master Mix, NEB), purified by SPRI beads (Agencourt AMPure XP, Beckman Coulter), and sequenced (HiSeq2000, Illumina).

For bioinformatics analysis of small RNA-seq data, the data were quantified and tested for differential expression with omiRas ${ }^{49}$. After raw data processing, the remaining tags were mapped to human genome 19 with bowtie (http://bowtie-bio.sourceforge.net/manual.shtml). Using various models of coding and noncoding RNAs retrieved from the UCSC table browser, the mapped tags were successfully annotated. Normalization and test for differential expression was performed as previously described $^{50}$. Those genes with false discovery rate (FDR) $<0.05$ and $|\log 2 \mathrm{fc}|>1.6$ were considered as differentially expressed genes (DEPs). For bioinformatics analysis of MACE data, all duplicate reads were removed from the raw datasets to remove any PCR bias. The preprocessing reads were aligned to the human genome with novoalign (http://www.novocraft.com). The RefSeq annotation track that includes mRNAs and lncRNAs (http://genome.ucsc. edu/cgi-bin/hgTables) was applied in annotations for genomic mapping positions. Normalization and test for differential expression were calculated as described for small RNAs. Those genes with FDR $<0.05$ and $|\log 2 \mathrm{fc}|>$ 1.6 were considered as DEPs. The functional classifications and pathway annotation of the DEPs were performed with the DAVID functional annotation tool (database for annotation, visualization, and integrated discovery) (http://david.abcc.ncifcrf.gov) using an enrichment cutoff of FDR $<0.05^{51}$.

\section{CRISPR/Cas9-mediated knockout of MALAT1}

The genomic sequence of MALAT1 was located at ensemble.org. Locate the exon or any exon of our interest (that may contain a functional domain, hot spot, etc.), copy and paste $23-500$ nt ( $200 \mathrm{nt}$ optimal) onto crispr. mit.edu to design CRISPR sgRNA strands for nuckase. Pick the top-ranked strands (with predicted faithfulness scores close to 100), to ligate into CRISPR constructs, at least two for nuclease. For every sgRNA strand, two oligos were designed and ordered (Supplementary Table 2), forward and reverse, complimentary to each other, with $5^{\prime}$ overhang CACC for one, AAAC for the other (sticky ends for the BbsI/BsmBI site). Perform the sub-cloning process: oligo insert annealing to form the oligo duplex, dilute the phosphorylated and annealed oligo duplexes 1:100 in $\mathrm{H}_{2} \mathrm{O}$, followed by the process of CRISPR Vector (pX462nickase-puro, donated by Dr. S. Paul Gao from Memorial Sloan Kettering Cancer Center) digestion and oligo insert ligation. Each construct was quickly transformed into Stbl3-competent cells, and at least six clones of each construct were selected for Sanger sequencing to validate insert ligation using the U6-Forward primer.

SURVEYOR primers are used to amplify a 500-800-bp fragment from the genomic sequence covering the sgRNA target site (Supplementary Table 2). HEK293T cells were plated onto a 24-well plate $\left(1.5 \times 10^{5}\right.$ cells/well $)$ for transfection with the above-constructed CRISPR vectors. Using harvested HEK293T cells $72 \mathrm{~h}$ post transfection, we used extracted genomic DNA with the help of Quick Extract, and the gDNA was ready for high-fidelity PCR. Three microliters were run on a 1.5-2.0\% gel to confirm a single band, correct-sized PCR product. The PCR product was purified using Qiagen DNA purification kit. The DNA was nanodropped to get the concentration, and normalized to $20 \mathrm{ng} / \mu \mathrm{l}$ with $\mathrm{ddH}_{2} \mathrm{O}$. The DNA heteroduplex was annealed for its formation and we performed Surveyor Nuclease S Assay. Half of the Nuclease $S$ treated sample was loaded on a $2 \%$ agarose gel, smaller digested products with intended sizes. To increase the targeted loss-of-function pooled screens, the lentiviral delivery systems for CRISPR screening were applied. The 
LentiCRISPR v2 plasmid deposited by Sanjana et al. was originally obtained from Addgene (a gift from Dr. S. P. Gao, MSKCC). Oligonucleotides targeting MALAT1 (only one exon) were subcloned into LentiCRISPR v2. Lentivirus was produced by transfecting these constructs together with psPAX2 and pMD2.G (Addgene) into HEK293T cells, and LoVo CRC cells were infected. Puromycin was used to weed out the CRISPR negative cells and the isogenic single-cell clones were obtained in a 96well plate using serial dilution method. Single clones of transduced cells were screened for indels in MALAT1 coding sequence by locus PCR/Sanger sequencing, and RT-PCR. Those without full-length MALAT1 expression were used to perform further experiments.

\section{Construction of the expression vectors}

The MALAT1 fragment (5'end 1020-nt sequences of the 8708-nt full-length MALAT1) containing the two predicted binding sites (the regions "170-178" and "729-737" in the 1020-nt MALAT1 fragment) for miR$15 \mathrm{~s}$, was subcloned into the pcDNA3.1 vector (Invitrogen), named pcDNA3.1-MALAT1 (Supplementary Table 3). The pcDNA3.1-MALAT1-mut (miR-15s) with point mutations in the miR-15s response elements (170-178 and 729-737) was also constructed. The plasmids of pcDNA3.1-MS2, pcDNA3.1-MS2-MALAT1, and pcDNA3.1-MS2-MALAT1-mut (miR-15s) were constructed for RNA immunoprecipitation (RIP) experiments. Human PTBP2 and RUNX2 genes were amplified by RT-PCR and subcloned into the expression vector pcDNA3.1, named pcDNA3.1-PTBP2 and pcDNA3.1RUNX2, respectively (Supplementary Table 3 ).

The 1020-nt region at the $5^{\prime}$ end of either MALAT1 or MALAT1-mut (miR-15s) was amplified using PCR and subcloned into the PmeI and Xhol sites of pmirGLO vector (Promega, Madison, WI) for luciferase reporter assay (Supplementary Table 3). The previously constructed recombinant plasmid pGL3-LEF/TCF promoter, control-positive plasmid pRL-SV40, and control-negative plasmid pGL3-MALAT1 promoter were also applied in our experiment. The predicted binding sites in the $3^{\prime}$ untranslated regions ( $3^{\prime}$ UTR) of the potential target genes of LRP6 (wild type, WT) and the mutant-type (MT) 3' UTR of LRP6 were synthesized and cloned into the pmirGLO vector, respectively. The plasmids pR-F, pRrunx2F, and pRVEGFaF, pRcox-2F were constructed as below: the entire $213 \mathrm{bp}$ of the runx2-5'UTR, $540 \mathrm{bp}$ of the VEGFa-5'UTR, and $140 \mathrm{bp}$ of the cox-2-5'UTR were chemically synthesized (containing the restriction enzyme cutting sites: NdeI and EcoRI) and cloned into pR-F between the NdeI and EcoRI sites, respectively. To generate the deletion mutants of $5^{\prime}$ UTR of runx2 and VEGFa, the mutant DNA fragments were also chemically synthesized and subcloned into the pR-F vector, named pRrunx2del1F, pRrunx2del2F, pRrunx2delPPTF, pRVEGFadel1F, pRVEGFadel2F, and pRVEGFadelPPTF. The $5^{\prime}$ UTR sequences of RUNX2, VEGFa, and COX-2 were shown in Supplementary Table 4.

\section{Cell transfection and lentivirus infection}

Transient transfections were performed using the Lipofectamine 2000 kit (Invitrogen) according to the manufacturer's instructions. The sequences of doublestranded microRNA mimics and their respective negative control RNAs (GeneChem, Shanghai, China) were listed in Supplementary Table 5. To obtain cell lines stably expressing MALAT1, MALAT1-mut (miR-15s), LoVo, or SW620 cells were transfected with the plasmid pcDNA3.1-MALAT1 or pcDNA3.1-MALAT1-mut (miR15s) using Lipofectamine 2000 kit as above, and selected with neomycin $(800 \mu \mathrm{g} / \mathrm{ml})$ for 4 weeks. Recombinant lentiviruses containing pre-hsa-miR-195 or the control were purchased from GeneChem (Shanghai, China). Recombinant lentiviruses containing pLV4-shRNA/LRP6, pLV4-shRNA/RUNX2, pLV4-shRNA/PTBP2, and pLV4shRNA/NT were constructed, respectively, and the suitable shRNA sequences for LRP6 were listed in Supplementary Table 6. Recombinant lentiviruses containing pLV4-MALAT1, pLV4-MALAT1-mut (miR-15s), or the control were prepared as previously described. LoVo or SW620 cells were infected with $2 \times 10^{6}$ transducing units of MALAT1, MALAT1-mut (miR-15s), or miR-195 overexpression lentiviruses and were selected with $2 \mu \mathrm{g} /$ $\mathrm{ml}$ puromycin for 2 weeks.

\section{RIP analysis}

LoVo cells were co-transfected with pMS2-GFP (Addgene) and pcDNA3.1-MS2, pcDNA3.1-MS2-MALAT1, and pcDNA3.1-MS2-MALAT1-mut (miR-15s), respectively. After $48 \mathrm{~h}$, cells were used to perform RNA immunoprecipitation (RIP) experiments using a GFP antibody (Roche) and the Magna RIP ${ }^{\mathrm{mix}}$ RNA-Binding Protein IP Kit (EMD Millipore, Billerica, Massachusetts, USA) according to the manufacturer's instructions. Briefly, LoVo cells were rinsed with ice-cold PBS, lysed using RIP lysis buffer. Magnetic beads binding the antibody of interest for IP were prepared. Mouse GFP antibody (Santa Cruz Biotechnology, Santa Cruz, CA, USA) and nonspecific mouse IgG antibody were used. IP of RNA-binding protein-RNA complex started by incubating the RIP lysates and magnetic beads binding the antibody of interest, together with rocking overnight at $4{ }^{\circ} \mathrm{C}$. Each IP was digested with proteinase $\mathrm{K}$ and $10 \%$ SDS at $55^{\circ} \mathrm{C}$ for 30 min after washing with RIP washing buffer. After incubation, centrifugation was used to obtain the supernatant, followed by washing and adding phenol:chloroform: isoamyl alcohol (125:24:1) to separate the phases. The aqueous phase was separated by adding chloroform and RNA was precipitated from the aqueous phase using $80 \%$ ethanol. 
Isolated RNA was treated with DNase I to remove any genomic DNA contamination. Each sample was reversetranscribed using the PrimeScript RT reagent Kit, followed by quantitative RNA analysis. All assays were performed in triplicate and independently repeated three times.

\section{RNA pulldown}

LncRNA-MALAT1 and lncRNA-MALAT1-mut (miR15s) were in vitro transcribed, respectively, from vectors pSPT19-MALAT1 and pSPT19-MALAT1-mut (miR15s), and biotin-labeled with the Biotin RNA Labeling Mix (Roche) and T7 RNA polymerase (Roche), treated with RNase-free DNase I (Roche), and purified with an RNeasy Mini Kit (Qiagen, Valencia, CA). One milligram of whole-cell lysates from LoVo cells were incubated with $3 \mu \mathrm{g}$ of purified biotinylated transcripts for $1 \mathrm{~h}$ at $25^{\circ} \mathrm{C}$, and the complexes were isolated with streptavidin agarose beads (Invitrogen). The RNA present in the pull-down material was detected by qRT-PCR analysis.

\section{Luciferase reporter assay}

Approximately 8000 HEK $293 \mathrm{~T}$ cells per well or 12,000 LoVo cells per well were plated into 96-well plates and were co-transfected with $50 \mathrm{nmol} / \mathrm{L}$ of $\mathrm{miR}-195 \mathrm{mimic}$ (or $\mathrm{NC}), 50 \mathrm{ng}$ of the luciferase reporter, and $5 \mathrm{ng}$ of the pRLCMV Renilla luciferase reporter using Lipofectamine 2000 kit (Invitrogen). After 48-h incubation, the Firefly and Renilla luciferase activities were quantified using a dual-luciferase reporter assay (Promega). Data are presented as the relative ratio of firefly luciferase activity to renilla luciferase activity.

\section{Quantitative real-time PCR}

Total RNA, including miRNA, was extracted using the TRIzol reagent (Invitrogen) according to the manufacturer's instructions. The concentrations of RNA were determined using a NanoDrop ND-1000 (NanoDrop), and aliquots of the samples were stored at $-80^{\circ} \mathrm{C}$. cDNA was synthesized with the PrimeScript RT Reagent Kit (TaKaRa) using $500 \mathrm{ng}$ of total RNA as the template. qPCR analyses were conducted to quantitate mRNA and IncRNA relative expression using SYBR Premix Ex Taq (TaKaRa) with GAPDH as an internal control. miRNA assays (Applied Biosystems) were used to determine the expression levels of miR-15s after reverse transcription by sequence-specific primers, and U6 small nuclear RNA was used as an internal control. The results of qPCR were defined from the threshold cycle $(\mathrm{Ct})$, and relative expression levels were calculated by using the $2^{-\triangle \triangle \mathrm{Ct}}$ method. PCR was performed using an ABI 7300 instrument (Applied Biosystems). The primers used for reverse transcription and PCR analysis were listed in Supplementary Tables 7 and 8.

\section{Western blot}

The frozen paired CRC tissues and NCTs or harvested cells were washed with cold PBS and then lysed in RIPA lysis buffer, and the lysates were cleared by centrifugation at $14,000 \mathrm{rpm}, 4{ }^{\circ} \mathrm{C}$ for $15 \mathrm{~min}$. Harvested proteins were first separated by $10 \%$ SDS-PAGE and then transferred to nitrocellulose membranes (Bio-Rad Laboratories). The membranes were blocked with 5\% nonfat milk and incubated with a mouse or rabbit first antibody at a dilution of 1:500 to $1: 1000$ (CST or Sigma). The membranes were subsequently incubated with a goat anti-mouse or goat anti-rabbit horseradish peroxidase secondary antibody (CST). The protein complex was detected using enhanced chemiluminescence reagents (Pierce). Actin was used as the internal control.

\section{Transwell analysis}

A total of $2.5 \times 10^{5}$ cells (in $100 \mu \mathrm{L}$ of F12K with $0.5 \%$ FCS) were seeded into the upper part of a transwell chamber (Transwell filter inserts in $6.5-\mathrm{mm}$ diameter, pore size of $8 \mu \mathrm{m}$, Corning Incorporated, NY, USA). In the lower part of the chamber, $600 \mu \mathrm{L}$ of F12K with $15 \%$ FCS and $10 \mu \mathrm{g} / \mathrm{ml}$ fibronectin was added and the assay was performed after $24 \mathrm{~h}$ at $37{ }^{\circ} \mathrm{C}$ and $5 \% \mathrm{CO}_{2}$. Migrated cells were analyzed by crystal violet staining, followed by observation under a DMI3000 B inverted microscope (Leica, Ernst-Leitz, Wetzlar, Germany). All assays were performed in triplicate and independently repeated three times.

\section{Tumor mouse model}

Experimental lung metastases were induced by injections of a single-cell suspension $\left(2 \times 10^{6}\right.$ cells in $\left.100 \mu \mathrm{l}\right)$ into the mouse lateral tail vein. To prevent pulmonary embolism caused by injected tumor cells, all cell injections were administered in a total volume of $500 \mu \mathrm{L}$ of PBS containing $0.1 \%$ BSA over a $60-\mathrm{s}$ duration ${ }^{52}$. Experimental liver metastases were achieved by intrasplenic injection with a single-cell suspension at $2 \times 10^{6}$ cells/injection site. For the hemi-spleen method, using a non-serrated hemostatic clamp, the spleen was clamped in the middle of its longitudinal axis, followed by the surgical split into two hemi-spleens, while both of the vascular pedicles were intact. Next, with a 31-Ga needle, the tumor cells were immediately injected into the clamped hemi-spleen. Nearly 2 min later, the needle was removed from the hemi-spleen, and the vascular pedicle draining the injected hemi-spleen was successfully ligated. Finally, using a scalpel, the injected hemi-spleen was excised, leaving a functional hemi-spleen free of tumor cells $^{53}$. Five to seven weeks later, the mice were anesthetized with pentobarbital sodium, and the lung or liver organs were excised, and the metastatic lesions were 
determined by hematoxylin-eosin (HE) staining. All the animal experiments were carried out with the approval of the Institutional Committee for Animal Research and were in accordance with national guidelines for the care and use of laboratory animals.

\section{Immunofluorescence staining}

Cells were fixed with methanol, blocked with 5\% BSA. The cells were first stained with $\beta$-catenin rabbit antibody followed by Cy3-conjugated goat anti-rabbit IgG (Millipore). Nuclear staining was done with DAPI (40, 6-diamidino-2-phenylindole). Cells were imaged with a TCS SP2 spectral confocal system (Leica, Ernst-Leitz, Wetzlar, Germany). All experiments were conducted according to instructions from the antibody manufacturer.

\section{Immunohistochemical staining}

Tissue arrays were constructed using the 124 paired $\mathrm{CRC}$ tissues and metastatic tissues. Immunohistochemical staining was performed on 4-mm sections of paraffinembedded tissues to determine the expression level of proteins. In brief, the slides were incubated in the first antibody (CST) diluted $1: 200$ at $4{ }^{\circ} \mathrm{C}$ overnight. The subsequent steps were performed using the EnVision FLEX High $\mathrm{pH}$ visualization system according to the manufacturer's instructions (Dako).

\section{Subcellular fractionation}

For the whole-cell proteins, the harvested cells were washed with cold PBS and then lysed in RIPA lysis buffer, and the lysates were cleared by centrifugation at $14,000 \mathrm{rpm}, 4{ }^{\circ} \mathrm{C}$ for $15 \mathrm{~min}$. For cytosolic and nuclear fractions, cells were first lysed with a buffer containing $10 \mathrm{mM}$ Tris- $\mathrm{HCl}, \mathrm{pH} 7.4,100 \mathrm{mM} \mathrm{NaCl}, 2.5 \mathrm{mM} \mathrm{MgCl}$, and $40 \mu \mathrm{g} / \mathrm{ml}$ digitonin for $15 \mathrm{~min}$, and the lysates were centrifuged at $2060 \times g$ for $15 \mathrm{~min}$ at $4{ }^{\circ} \mathrm{C}$. The supernatant was just the cytosolic fraction. Subsequently, the pellets were washed, incubated with RIPA buffer at $4{ }^{\circ} \mathrm{C}$ for $15 \mathrm{~min}$, and the nuclear fraction was collected after centrifugation at $4{ }^{\circ} \mathrm{C}$ for $30 \mathrm{~min}$ at $21,000 \times \mathrm{g}$.

\section{Polysome analysis}

Each cell sample was pre-incubated with $100 \mu \mathrm{g} / \mathrm{ml}$ cycloheximide (Sigma) for $15 \mathrm{~min}$, and the cytoplasmic lysates were prepared and fractionated by ultracentrifugation on $15-60 \%$ linear sucrose gradients. For each sample, 10 fractions were collected, and the RNA was extracted from each fraction for real-time PCR analysis as described by Yoon et al. ${ }^{54}$.

\section{Northern blotting}

Twenty micrograms of total RNA from CRC cells were run in agarose/formaldehyde gels. After transferring to nylon membranes, MALAT1 and GAPDH mRNA were detected using antisense oligomer probes (MALAT1 KO Probe 1: CCTTCCGCCGCCTTTGTGAG (1198-1218 nt), MALAT1 KO Probe 2: CTGAGCCCCAGCCTTGCAGG GACGGTTGAGAAGTG (831-865 nt), MALAT1 KO Probe 3: CTAAGATAGCAGCACAACTCGTCGCTGC GTCCCAA (151-185 nt), MALAT1 KO Probe 4: GAAATCGGAGCAGCACGGGCTGTCTGCTTGGGAA A (711-745 nt), and GAPDH probe: GCCCAATACGA CCAAATCC, respectively) that were end-labeled with ${ }^{32} \mathrm{P}$. All the bands were visualized using a Typhoon 9400 scanner (GE Healthcare, USA). All the procedures was performed as previously described ${ }^{54}$.

\section{Statistical analysis}

The results were presented as the mean values \pm SEM. Overall survival (OS) rates and disease-free survival (DFS) rates were calculated actuarially according to the Kaplan-Meier method with log-rank test and were measured from the day of surgery. Receiver-operating characteristic (ROC) curve and the areas under ROC curve (AUC) were constructed to evaluate the diagnostic value of gene detection. Differences between groups were estimated using the $\chi^{2}$ test, the Student $t$ test, the Mann-Whitney $U$ test, and the repeated-measures ANOVA test. Relationships were explored by the Spearman correlation. A value of $P<0.05$ was considered statistically significant. SPSS 20.0 and Graphpad prism 5.0 were used for statistical analyses and scientific graphing.

\section{Study approval}

All animal protocols were approved by the Animal Care Commission of Shanghai University of Traditional Chinese Medicine. All procedures involving human tumor biopsies were performed with the approval of the ethics committee of the Shuguang Hospital, Shanghai University of Traditional Chinese Medicine on samples from patients who had given written informed consent.

\section{Acknowledgements \\ This work was supported by National Natural Science Foundation of China (No. 81520108031 , No. 81830120 , and No. 81473478 to Q.L.), National Natural Science Foundation of China (No. 81303102, No. 81573749 to Q.J.), National Natural Science Foundation of China (No. 81303103, No. 81673783 to X.L.), the Science Foundation of Shanghai Committee of Science Project (No. \\ 14430722900 to Q.L.), the Program for Outstanding Medical Academic Leader and Shanghai Academic Research Leader (No. 16XD1403600 to Q.L.), Shanghai Municipal Education Commission (No. 13 CG47 to X.L.), and Shanghai Rising- Star Program (No. 18QA1404100 to X.L.).}

\section{Author contributions}

Q.J., G.C., X.L., and Q.L. conceived and designed the study and the experimental setup and wrote the paper. Q.J., X.L., Y.Z., Y.W., L.Z., and H.S. performed the experiments. G.C. and Q.L. analyzed the data. All authors read and approved the final version of the paper.

Conflict of interest

The authors declare that they have no conflict of interest. 


\section{Publisher's note}

Springer Nature remains neutral with regard to jurisdictional claims in published maps and institutional affiliations.

Supplementary Information accompanies this paper at (https://doi.org/ 10.1038/s41419-019-1598-x).

\section{Received: 20 November 2018 Revised: 12 April 2019 Accepted: 12 April} 2019

Published online: 16 May 2019

\section{References}

1. Siegel, R. L., Miller, K. D. \& Jemal, A. Cancer statistics, 2017. CA Cancer J. Clin. 67, 7-30 (2017).

2. Ribatti, D., Mangialardi, G. \& Vacca, A. Stephen Paget and the "seed and soil" theory of metastatic dissemination. Clin. Exp. Med. 6, 145-149 (2006).

3. Batista, P. J. \& Chang, H. Y. Long noncoding RNAs: cellular address codes in development and disease. Cell 152, 1298-1307 (2013).

4. Li, L. et al. Role of human noncoding RNAs in the control of tumorigenesis. Proc. Natl Acad. Sci. USA 106, 12956-12961 (2009).

5. Gupta, R. A. et al. Long non-coding RNA HOTAIR reprograms chromatin state to promote cancer metastasis. Nature 464, 1071-1076 (2010).

6. Ji, P. et al. MALAT-1, a novel noncoding RNA, and thymosin beta4 predict metastasis and survival in early-stage non-small cell lung cancer. Oncogene 22 8031-8041 (2003)

7. Gutschner, T., Hämmerle, M. \& Diederichs, S. MALAT1-a paradigm for long noncoding RNA function in cancer. J. Mol. Med. 91, 791-801 (2013).

8. Tripathi, $V$. et al. The nuclear-retained noncoding RNA MALAT1 regulates alternative splicing by modulating SR splicing factor phosphorylation. Mol. Cell 39, 925-938 (2010).

9. Tripathi, V. et al. Long noncoding RNA MALAT1 controls cell cycle progression by regulating the expression of oncogenic transcription factor B-MYB. PLOS Genet. 9, e1003368 (2013).

10. Hirata, $\mathrm{H}$. et al. Long noncoding RNA MALAT1 promotes aggressive renal cell carcinoma through Ezh2 and Interacts with miR-205. Cancer Res. 75 1322-1331 (2015).

11. Latorre, E. et al. The ribonucleic complex HuR-MALAT1 represses CD133 expression and suppresses epithelial-mesenchymal transition in breast cancer. Cancer Res. 76, 2626-2636 (2016)

12. Wilusz, J. E., Freier, S. M. \& Spector, D. L. 3'end processing of a long nuclearretained noncoding RNA yields a tRNA-like cytoplasmic RNA. Cell 135, 919-932 (2008)

13. Ji, Q. et al. Long non-coding RNA MALAT1 promotes tumor growth and metastasis in colorectal cancer through binding to SFPQ and releasing oncogene PTBP-2 from SFPQ/PTBP-2 complex. Br. J. Cancer 111, 736-748 (2014).

14. Zheng, H. T. et al. High expression of IncRNA MALAT1 suggests a biomarker of poor prognosis in colorectal cancer. Int. J. Clin. Exp. Pathol. 7, 3174-3181 (2014).

15. Ji, Q. et al. Resveratrol Inhibits invasion and metastasis of colorectal cancer cells via MALAT1 mediated Wnt/ß-catenin signal pathway. PLOS ONE 8, e78700 (2013).

16. Lu, $\mathrm{H}$. et al. Long non-coding RNA MALAT1 modulates radiosensitivity of HRHPV+ cervical cancer via sponging miR-145. Tumour Biol. 37, 1683-1691 (2016).

17. $Y u, F$. et al. MALAT1 functions as a competing endogenous RNA to mediate Rac1 expression by sequestering miR-101b in liver fibrosis. Cell Cycle 14, 3885-3896 (2015).

18. Chou, J. et al. MALAT1 induced migration and invasion of human breast cancer cells by competitively binding miR-1 with cdc42. Biochem. Biophys. Res. Commun. 472, 262-269 (2016).

19. Wang, S. H. et al. The IncRNA MALAT1 functions as a competing endogenous RNA to regulate MCL-1 expression by sponging miR-363-3p in gallbladder cancer. J. Cell Mol. Med. 20, 2299-2308 (2016).

20. Luan, W. et al. Long non-coding RNA MALAT1 acts as a competing endogenous RNA to promote malignant melanoma growth and metastasis by sponging miR-22. Oncotarget 7, 63901-63912 (2016).

21. Tay, Y., Rinn, J. \& Pandolfi, P. P. The multilayered complexity of ceRNA crosstalk and competition. Nature 505, 344-352 (2014).
22. Chandradoss, S. D., Schirle, N. T., Szczepaniak, M., MacRae, I. J. \& Joo, C. A dynamic search process underlies microRNA targeting. Cell 162, 96-107 (2015).

23. Nishi, $K$. et al. Control of the localization and function of a miRNA silencing component TNRC6A by Argonaute protein. Nucleic Acids Res. 43, 9856-9873 (2015).

24. Schraivogel, D. et al. Importin- $\beta$ facilitates nuclear import of human GW proteins and balances cytoplasmic gene silencing protein levels. Nucleic Acids Res. 43, 7447-7461 (2015).

25. Weinmann, L. et al. Importin 8 is a gene silencing factor that targets argonaute proteins to distinct mRNAs. Cell 136, 496-507 (2009).

26. Leucci, E. et al. microRNA-9 targets the long non-coding RNA MALAT1 for degradation in the nucleus. Sci. Rep. 3, 2535 (2013).

27. Zhao, J. J. et al. miR-30-5p functions as a tumor suppressor and novel therapeutic tool by targeting the oncogenic Wnt/ß-catenin/BCL9 pathway. Cancer Res. 74, 1801-1813 (2014).

28. Huang, S. M. et al. Tankyrase inhibition stabilizes axin and antagonizes Wnt signaling. Nature 461, 614-620 (2009).

29. Guo, Z. J. et al. Transcription factor RUNX2 up-regulates chemokine receptor CXCR4 to promote invasive and metastatic potentials of human gastric cancer. Oncotarget 7, 20999-21012 (2016).

30. Baniwal, S. K. et al. Runx2 transcriptome of prostate cancer cells: insights into invasiveness and bone metastasis. Mol. Cancer 9, 258 (2010).

31. Kafasla, P., Morgner, N., Robinson, C. V. \& Jackson, R. J. Polypyrimidine tractbinding protein stimulates the poliovirus IRES by modulating elF4G binding EMBO J. 29, 3710-3722 (2010).

32. Cornelis, S., Tinton, S. A., Schepens, B., Bruynooghe, Y. \& Beyaert, R. UNR translation can be driven by an IRES element that is negatively regulated by polypyrimidine tract binding protein. Nucleic Acids Res. 33, 3095-3108 (2005).

33. Schepens, B., Tinton, S. A., Bruynooghe, Y., Beyaert, R. \& Cornelis, S. The polypyrimidine tract-binding protein stimulates HIF-1alpha IRES-mediated translation during hypoxia. Nucleic Acids Res. 33, 6884-6894 (2005).

34. Stoneley, M. et al. Analysis of the c-myc IRES; a potential role for cell-type specific trans-acting factors and the nuclear compartment. Nucleic Acids Res. 28, 687-694 (2000)

35. Arcondéguy, T., Lacazette, E., Millevoi, S., Prats, H. \& Touriol, C. VEGF-A mRNA processing, stability and translation: a paradigm for intricate regulation of gene expression at the post-transcriptional level. Nucleic Acids Res. 41, 7997-8010 (2013).

36. Huang, J. F. et al. Hepatitis B virus X protein ( $\mathrm{HBX}$ )-related long noncoding RNA (IncRNA) downregulated expression by $\mathrm{HBx}$ (Dreh) inhibits hepatocellular carcinoma metastasis by targeting the intermediate filament protein vimentin. Hepatology 57, 1882-1892 (2013).

37. Yuan, J. H. et al. A long noncoding RNA activated by TGF- $\beta$ promotes the invasion-metastasis cascade in hepatocellular carcinoma. Cancer Cell $\mathbf{2 5}$ 666-681 (2014)

38. Kim, J. et al. Long noncoding RNA MALAT1 suppresses breast cancer metastasis. Nat. Genet. 50, 1705-1715 (2018).

39. Furió-Tarí, P., Tarazona, S., Gabaldón, T., Enright, A. J. \& Conesa, A. spongeScan: A web for detecting microRNA binding elements in IncRNA sequences. Nucleic Acids Res. 44, W176-W180 (2016).

40. Tamai, K. et al. LDL-receptor-related proteins in Wnt signal transduction. Nature 407, 530-535 (2000)

41. Li, Y., Lu, W., He, X., Schwartz, A. L. \& Bu, G. LRP6 expression promotes cancer cell proliferation and tumorigenesis by altering beta-catenin subcellular distribution. Oncogene 23, 9129-9135 (2004).

42. Liu, C. C., Prior, J., Piwnica-Worms, D. \& Bu, G. LRP6 overexpression defines a class of breast cancer subtype and is a target for therapy. Proc. Natl Acad. Sci. USA 107, 5136-5141 (2010).

43. Zhang, J., Li, Y., Liu, Q., Lu, W. \& Bu, G. Wnt signaling activation and mammary gland hyperplasia in MMTV-LRP6 transgenic mice: implication for breast cancer tumorigenesis. Oncogene 29, 539-549 (2011).

44. Fahmy, K. et al. Myoferlin plays a key role in VEGFA secretion and impacts tumor-associated angiogenesis in human pancreas cancer. Int. J. Cancer $\mathbf{1 3 8}$ 652-663 (2016)

45. Llovet, J. M. Focal gains of VEGFA: candidate predictors of sorafenib response in hepatocellular carcinoma. Cancer Cell 25, 560-562 (2014).

46. Shi, $Y$. et al. Therapeutic potential of targeting IRES-dependent c-myc translation in multiple myeloma cells during ER stress. Oncogene 35, 1015-1024 (2016) 
47. Weingarten-Gabbay, S. et al. The translation initiation factor DAP5 promotes IRES-driven translation of p53 mRNA. Oncogene 33, 611-618 (2014).

48. Müller, S. et al. APADB: a database for alternative polyadenylation and microRNA regulation events. Database (Oxford). https:/doi.org/10.1093/ database/bau076 (2014).

49. Müller, S. et al. OmiRas: a Web server for differential expression analysis of miRNAs derived from small RNA-Seq data. Bioinformatics 29, 2651-2652 (2013).

50. Anders, S. \& Huber, W. Differential expression analysis for sequence count data. Genome Biol. 11, R106 (2010).
51. Huang, D. W. et al. DAVID bioinformatics resources: expanded annotation database and novel algorithms to better extract biology from large gene lists. Nucleic Acids Res. 35, W169-W175 (2007).

52. Minn, A. J. et al. Genes that mediate breast cancer metastasis to lung. Nature 436, 518-524 (2005).

53. Jain, A. et al. Synergistic effect of a granulocyte-macrophage colony stimulating factor-transduced tumor vaccine and systemic interleukin-2 in the treatment of murine colorectal cancer hepatic metastases. Ann. Surg. Oncol 10 810-820 (2003).

54. Yoon, J. H. et al. LincRNA-p21 suppresses target mRNA translation. Mol. Cell. 47 648-655 (2012) 